\title{
Glycaemic benefit of structured diabetes education in Nepal, a resource limited environment
}

\author{
JAYA PRADHAN, ${ }^{1}$ SATYAN M RAJBHANDARI ${ }^{2}$
}

\begin{abstract}
Introduction: Structured diabetes education is a key element in the management of type 2 diabetes, but this is challenging to deliver in settings where resources are limited.

Methods: We conducted a randomised evaluation of a single 90-minute session of structured diabetes education added to the local standard of diabetes care compared with a control group (standard diabetes care only) in 150 patients with recently diagnosed type 2 diabetes in Nepal. The level of knowledge about diabetes was low.

Results: Follow-up 6 months after the intervention showed that the reduction in mean (SD) fasting plasma glucose was significantly larger in the education group (from 8.6 (2.9) $\mathrm{mmol} / \mathrm{L}$ to $6.7(1.2) \mathrm{mmol} / \mathrm{L})$ compared with the control group (from 8.1 (1.8) $\mathrm{mmol} / \mathrm{L}$ to 7.0 (1.8) $\mathrm{mmol} / \mathrm{L})(p=0.029$ for comparison between groups). A significant reduction in postprandial plasma glucose also occurred in the education group (from 11.7 (3.7) $\mathrm{mmol} / \mathrm{L}$ to $8.3(1.2) \mathrm{mmol} / \mathrm{L}$ ) compared with the control group (from 11.5 (4.0) $\mathrm{mmol} / \mathrm{L}$ to 9.7 (2.3) $\mathrm{mmol} / \mathrm{L})(\mathrm{p}=0.005$ between groups). $A$ trend to reduced $\mathrm{HbA}_{1 \mathrm{c}}$ was seen for the education versus the control group at 6 months $(p=0.06)$. There were no significant changes in lipids or blood pressure. Overall energy intake and the proportion of energy intake from fat was lower at 6 months compared with baseline for the education group but not for the control group, although there were no significant changes in anthropometric parameters.

Conclusion: Our results suggest that a single session of structured diabetes education may provide glycaemic benefits in newly-diagnosed type 2 diabetes patients, and that this may be a pragmatic means of improving diabetes self-care in resource-limited countries such as Nepal.

Br J Diabetes 2020;20:107-112
\end{abstract}

\footnotetext{
Professor and Head of Department, Central Department of Home Science, Padmakanya Campus, Tribhuvan University, Kathmandu, Nepal

2 Consultant Physician and Honorary Clinical Professor, Lancashire Teaching Hospital, Chorley, Lancashire, UK
}

Address for correspondence: Dr Satyan M Rajbhandari Consultant Physician, Lancashire Teaching Hospital, Honorary Clinical Professor, University of Central Lancashire, Chorley \& South Ribble Hospital, Preston Road, Chorley PR7 1PP, UK

E-mail: satyan.rajbhandari@|thtr.nhs.uk

https://doi.org/10.15277/bjd.2020.260
Key words: type 2 diabetes, structured diabetes education, diabetes self-management, fasting plasma glucose

\section{Introduction}

Weight gain is the key element of the pathophysiology of type 2 diabetes in susceptible individuals. In particular, interventions that promote weight loss reduce the likelihood of progression from prediabetic states to type 2 diabetes, and contribute to improved control of glycaemia in patients who already have type 2 diabetes. ${ }^{1}$ Lifestyle interventions, including structured self-management education on nutrition and other aspects of a healthy lifestyle, are a key element of management guidelines in this area, with proven benefits in terms of improved metabolic outcomes. ${ }^{2-4}$

The application of successful diabetes self-management education depends critically on the availability of resources to deliver it. Developing countries may lack the resources to provide such interventions, or people may face barriers to implementing lifestyle advice such as cultural or family issues or limited access to healthy foods, among others. ${ }^{5,6} \mathrm{Nepal}$ is a low-income developing country with an increasing burden of diabetes. A systematic review of studies evaluating the prevalence of diabetes found a pooled prevalence of $8 \%$, with an eight-fold higher prevalence in the urban compared with the rural setting. ${ }^{7}$ A more recent cross-sectional survey found a prevalence of diabetes of $11.7 \%{ }^{8}$

Weight loss is the mainstay of treatment or even reversal of diabetes for which a very low calorie diet is needed..$^{9}$ The role of a low glycaemic index diet has not been fully established but in meta-analysis it has been shown to have some effect. ${ }^{10}$ Nepalese people usually eat two main meals a day around mid-morning and late evening with one or two small snacks in between meals. 'Dal-Bhat' (rice, pulses and vegetable curry) is the traditional diet in most parts of the country consumed twice a day and is high in carbohydrate of intermediate glycaemic index. The use of high calorie fast food is limited; however, there is increasing use of sugary drinks. Therefore, dietary intervention could be an easier target to improve diabetes control in Nepal. Physical activity is also important for diabetes control as it reduces insulin resistance, ${ }^{11}$ which is a common pathophysiology for type 2 diabetes. Modification of diet and increased physical activity are needed to improve control of diabetes. Good diabetes knowledge and continual support are needed for long-term behavioural changes to maintain these benefits of non-medical interventions for diabetes. 
However, diabetes knowledge is limited among type 2 diabetes patients in Nepal ${ }^{12}$ and there is no national provision of support or of structured diabetes education. We evaluated the effectiveness of a single session of structured diabetes nutritional education in improving glycaemic control in a randomised controlled trial in newly-diagnosed type 2 diabetes patients in Nepal.

\section{Methods}

Patients eligible for the study were men or women (any ethnicity) with type 2 diabetes diagnosed within the previous year who were aged $\geq 30$ years and $<60$ years and were managed in one of five diabetes clinics in an urban area of Kathmandu, Nepal. Key exclusion criteria were type 1 diabetes (defined as requiring insulin treatment within 6 months of diagnosis), severe and enduring mental health problems, the presence of other chronic conditions which require dietary restriction (such as chronic renal failure), or other serious conditions that could impair participation in the study.

Thirty patients were selected from each diabetes management centre. After providing written informed consent to participate, in-depth interviews supported by a custom-designed questionnaire were used to gather information. The baseline questionnaire consists of four sections. The first part of the questionnaire covered the patient's socioeconomic and demographic information; the second part covered knowledge about diabetes, family history, medical expenses and anthropometric, biological and clinical status; the third part covered lifestyle and related behaviour such as exercise, alcohol consumption and smoking; and the last part of the questionnaire assessed the existing dietary practice and dietary pattern. This section assessed the calorie consumption pattern, reasons for restriction of particular food, nutritional knowledge, frequency of meals and attitudes towards life after being diagnosed with diabetes. A 24-hour dietary recall and food frequency questionnaire were also used to explore the previous 24 hours of food intake. These were validated in 10 people with type 2 diabetes beforehand by in-depth interview in order to evaluate the suitability of the contents, clarity and sequence of the questionnaire, the feasibility and reliability of data collection.

Patients were randomised to the educational intervention or to a control group who received the local standard of care for the management of diabetes without structured education. In practice, some patients who consented to participate in the study then declined the dietary education; these patients were included in the control group. The education intervention lasted 90 minutes and contained material designed to improve the understanding of daily calorie needs, estimation of calories as per need of an individual, ratio of calories from macronutrients, five food groups, dietary source of macronutrients, proportion and size, meal times and meal distribution and meal planning.

Individualised diet plans gave priority to food exchange system, the portion size of each serving, hand portion of different food groups, importance of each food group and its serving numbers, frequency of whole day meals, importance of healthy snacks, cooking methods, etc. Estimation of individual calorie requirements was calculated on the basis of the respondent's height, weight and occupation according to the guidelines of the Indian Council for Medical Research.

Patients were followed up 6 months later for determination of glycaemic parameters, which included both fasting and 2-hour postprandial blood glucose along with $\mathrm{HbA}_{1 c}$. Patients were visited in their homes for completion of a questionnaire at this time to avoid loss to follow-up. Nutritional analysis was carried out on reported dietary intake of each case manually by the same researcher using knowledge of the local dietary pattern. Data were analysed using summary statistics and independent sample t-tests were used for some parameters to determine the statistical significance of changes associated with the intervention.

Ethical approval was obtained from University Grants Commission of Nepal as it was part of the PhD project.

\section{Results}

\section{Subjects at baseline}

The education and control groups were well matched at baseline for mean age (47 vs 48 years) and the proportion of men/ women ( $43 \% / 57 \%$ vs $47 \% / 53 \%)$.

Knowledge about diabetes was patchy at baseline. For example, most subjects understood that diabetes is concerned with high blood glucose ( $83 \%$ of the intervention group and $78 \%$ of the control group), but $11 \%$ or fewer of the intervention group and $6 \%$ or fewer of the control group could answer questions correctly concerning risk factors for type 2 diabetes (age, insulin deficiency, sedentariness, overweight, excessive sugar intake). Similarly, $39 \%$ of the intervention group and $54 \%$ of the control group were unaware of glycaemic thresholds for fasting plasma glucose or postprandial glucose. About $40 \%$ in each group knew that diabetes is a lifelong disease,

Most of the intervention group (93\%) and the control group (81\%) knew that diabetes can be managed with diet but fewer knew that exercise (60\% vs $36 \%$ ) or medication ( $75 \%$ vs $61 \%$ ) could be used for this purpose. When asked why they needed to manage their diabetes, the most common multiple choice response selected was "As suggested by physician" (32\% and $54 \%$, respectively), followed by "to control blood glucose level" (28\% and $22 \%$, respectively), "to live long life" (17\% in each group) and "to protect other vital organs" (17\% and 6\%, respectively).

\section{Effects on metabolic parameters}

Mean fasting plasma glucose and 2-hour postprandial glucose fell significantly over the 6 months following the educational intervention compared with the control group (Table 1). Trends towards improvements in $\mathrm{HbA}_{1 \mathrm{c}}$ for the intervention group compared with the control group almost achieved statistical significance ( $p=0.06$ in each case).

Body mass index declined slightly in the intervention group and increased slightly in the control group, but differences between groups did not approach statistical significance (Table 1). There were no significant effects on other anthropometric 
Table 1 Mean changes in metabolic parameters at baseline and at 6 months after a single dietary education intervention

\begin{tabular}{|c|c|c|c|c|c|}
\hline & \multicolumn{2}{|c|}{ Education intervention $(n=60)$} & \multicolumn{2}{|c|}{ Control group $(n=90)$} & \multirow[t]{2}{*}{$P$ value } \\
\hline & Baseline & 6 months & Baseline & 6 months & \\
\hline \multicolumn{6}{|l|}{ Glycaemic parameters } \\
\hline FPG $(\mathrm{mmol} / \mathrm{L})$ & $8.6(2.9)$ & $6.7(1.2)$ & $8.1(1.8)$ & $7.0(1.8)^{\star}$ & 0.029 \\
\hline PPG (mmol/L) & $11.7(3.7)$ & $8.3(1.2)$ & $11.5(4.0)$ & $9.7(2.3)$ & 0.005 \\
\hline $\mathrm{HbA}_{1 \mathrm{c}}(\%)$ & $8.9(2.1)$ & $7.8(1.5)$ & $8.7(2.4)$ & $8.1(1.2)$ & 0.06 \\
\hline \multicolumn{6}{|l|}{ Lipid parameters } \\
\hline LDL-cholesterol (mmol/L) & $3.2(1.6)$ & $2.8(0.7)$ & $3.0(1.0)$ & $2.9(0.9)$ & 0.121 \\
\hline Total cholesterol (mmol/L) & $4.5(0.8)$ & $4.3(0.6)$ & $4.4(0.9)$ & $4.4(0.9)$ & 0.07 \\
\hline Triglycerides (mmol/L) & $1.9(0.9)$ & $1.7(0.5)$ & $2.0(1.0)$ & $1.9(1.0)$ & 0.551 \\
\hline HDL-cholesterol (mmol/L) & $1.2(0.3)$ & $1.2(0.2)^{\star}$ & $1.1(0.2)$ & $1.1(0.2)^{\star}$ & 0.507 \\
\hline \multicolumn{6}{|l|}{ Blood pressure } \\
\hline Systolic (mmHg) & $124(15)$ & $120(13)$ & $122(15)$ & $121(14)$ & 0.175 \\
\hline Diastolic (mmHg) & $81(9)$ & $82(8)$ & $82(11)$ & $81(9)$ & 0.543 \\
\hline \multicolumn{6}{|c|}{ Anthropogenic parameters } \\
\hline Body mass index $\left(\mathrm{kg} / \mathrm{m}^{2}\right)$ & $25.8(3.8)$ & $25.4(3.9)$ & $26.4(3.6)$ & $27.0(6.8)$ & 0.275 \\
\hline Waist-hip ratio & $0.92(0.06)$ & $0.91(0.07)$ & $0.95(0.6)$ & $0.95(0.6)$ & 0.536 \\
\hline Waist circumference (cm) & $88.1(9.6)$ & $85.4(8.0)$ & $89.2(9.7)$ & $88.5(11.3)$ & 0.064 \\
\hline
\end{tabular}

parameters (waist circumference and waist-hip ratio), lipid parameters or blood pressures associated with the intervention (Table 1).

\section{Calorie intake}

Mean total energy intake decreased in the education group from 1972 to $1663 \mathrm{kcal}$ (men) and from 1618 to $1354 \mathrm{kcal}$ (women). Corresponding changes in energy intake in the control group were from 1744 to $1666 \mathrm{kcal}$ (men) and from 1493 to $1367 \mathrm{kcal}$ (women). The differences in energy intake were significant between groups ( $p<0.01$ for men and $p<0.04$ for women). Figure 1 shows the proportions of subjects whose energy intake was higher or lower than their individually estimated energy requirement. There was a more pronounced shift towards optimal (or lower) energy intake at 6 months in the education group than in the control group. Table 2 shows the energy intake from

Figure 1. Changes in mean calorie intake according to individualised estimated calorie requirements.

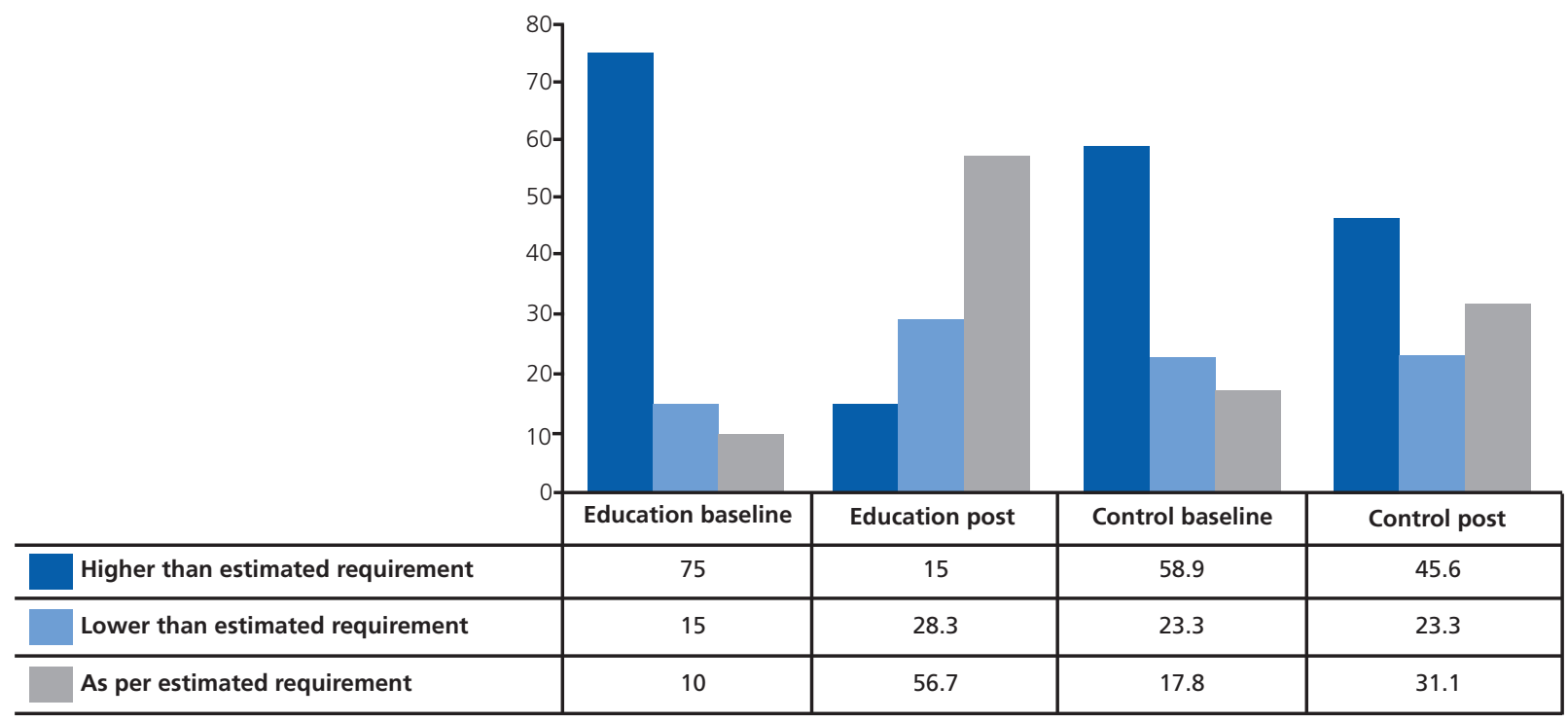


Table 2 Changes in calorie intake (percent of total daily energy intake) at baseline and 6 months after a single dietary education intervention

\begin{tabular}{|c|c|c|c|c|}
\hline & \multicolumn{2}{|c|}{$\begin{array}{l}\text { Educational } \\
\text { intervention } \\
\text { group }(n=60)\end{array}$} & \multicolumn{2}{|c|}{$\begin{array}{l}\text { Control group } \\
(n=90)\end{array}$} \\
\hline & Baseline & 6 months & Baseline & 6 months \\
\hline Calories from fat (\%) & 29 & 23 & 27 & 27 \\
\hline $\begin{array}{l}\text { Calories from } \\
\text { protein (\%) }\end{array}$ & 17 & 19 & 17 & 17 \\
\hline $\begin{array}{l}\text { Calories from } \\
\text { carbohydrate (\%) }\end{array}$ & 54 & 57 & 56 & 56 \\
\hline
\end{tabular}

Table 3 Changes in medications for diabetes at baseline and 6 months after a single dietary education intervention

\begin{tabular}{lll}
\hline & $\begin{array}{l}\text { Educational } \\
\text { intervention } \\
\text { group }(\mathbf{n = 6 0})\end{array}$ & $\begin{array}{l}\text { Control } \\
\text { group } \\
(\mathbf{n = 9 0 )}\end{array}$ \\
\hline No change in diabetes medications (\%) & 40 & 34 \\
Initiated diabetes medicine (\%) & 2 & 13 \\
Added new medicine (\%) & 13 & 11 \\
Increased dose (\%) & 0 & 24
\end{tabular}

macronutrients. Energy intake from fat was lower in the education group at 6 months relative to baseline. Energy intake in the control group was essentially unchanged at 6 months.

\section{Changes in medications for diabetes}

Similar proportions of the intervention group (50\%) and the control group (49\%) were taking medications for diabetes 6 months after the intervention (most subjects were initiated on metformin). More subjects in the control group added a new medicine for diabetes or increased the dose of their existing medicine than in the education group (Table 3 ). The proportions who added a new medicine were similar between the groups.

\section{Discussion}

We randomised 150 people with recently diagnosed type 2 diabetes in Nepal to a single structured education session or to a control group who received the local standard of care for diabetes. As this was done as part of a PhD programme in the Department of Nutrition, more emphasis was given to the dietary education. Due to limitation of resources, none of the groups received behavioural changes approach, which were frequently done in other studies. Our population was broadly typical of participants in clinical trials in type 2 diabetes; for example, $\mathrm{HbA}_{1 \mathrm{c}}$ at baseline was $8.2 \%$ in a large meta-analysis

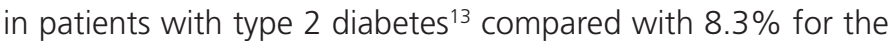
overall population in our study. Levels of LDL-cholesterol, total cholesterol and triglycerides were higher than the cut-off values for a diagnosis of dyslipidaemia in management guidelines, ${ }^{2,14}$ which is a common finding in populations with type 2 diabetes. ${ }^{15}$

Receipt of the intervention was associated with improved measures of plasma glucose 6 months later compared with the control group. Non-significant trends in $\mathrm{HbA}_{1 \mathrm{c}}$ and LDL-cholesterol and total cholesterol were also seen for the intervention group compared with controls. The level of knowledge and understanding concerning diabetes and its treatments were poor at baseline and improved somewhat after the intervention. The benefits observed in our study are consistent with previous demonstrations of improved metabolic outcomes following the application of structured diabetes education elsewhere. ${ }^{16-19}$ Intensification of medication regimens for diabetes by initiating a new medicine or increasing the dose of an existing medicine occurred more frequently in the control group than in the education group, while the proportions who initiated an additional medicine were similar in the two groups. Due to the lack of an insurance system or government funding in Nepal, patients have to purchase their own medications. Although we did not collect data on the exact cost, the reduced number of medications needed in the intervention group will have had a direct financial benefit to the patients. Thus, changes in medication for diabetes could indirectly account for the glycaemic benefits that we observed following the educational intervention. Overall, our data suggest that giving newly-diagnosed type 2 diabetes patients a single session of education may provide a pragmatic option for improving diabetes self-management which is practical for a severely resource-limited country like Nepal. Studies have shown that behavioural changes and associated improvements in biomedical outcomes are short lived. ${ }^{20}$ The long-term benefit of this intervention is not clear from our study.

We used a recall system for reporting and recording energy intake. Patients were asked to recall all foods and beverages consumed during the last 24-hour period before the interview both at the baseline and at 6 months. Due to limitation of resources, weighing of food or meal replacement was not considered. There was even a possibility of under-reporting or consuming a healthy diet before the follow-up visit. This could reflect on the lack of a significant change in body mass index during follow-up. At baseline the average body mass index of our patients was $25.8 \mathrm{~kg} / \mathrm{m}^{2}$ and $26.4 \mathrm{~kg} / \mathrm{m}^{2}$ in the education and control groups, respectively, which is not very high and thus will make it harder to lose weight. We did not use a behavioural changes approach or regular contacts as has been done in other studies due to resource limitation. Future studies should consider these approaches and concentrate on weight loss and its maintenance long term. Due to the design of our study we did not collect data on retention of diabetesspecific knowledge after 6 months, which would have given insight into the long-term effectiveness of the one-off educational intervention. We also did not assess the psychological outcome such as quality of life, diabetes distress and coping strategies due to lack of resources, which has been shown to be beneficial in other structured education intervention trials. ${ }^{18,21}$ Future clinical trials conducted in developing countries should take these into account when designing studies. 


\section{Key messages}

- Structured diabetes education is of proven efficacy for improving the self-management skills of people with diabetes

- Opportunities to deploy structured diabetes education are limited in resource-limited environments

- 150 Nepalese patients with type 2 diabetes were randomised to a single session of structured diabetes education (a control group received standard diabetes management)

- Fasting plasma glucose was significantly lower in the education intervention group compared with the controls 6 months later, with a strong trend to benefit for $\mathrm{HbA}_{1 \mathrm{c}}$ and postprandial plasma glucose

We encountered lots of challenges in conducting this study. First, we had to convince clinicians to allow patients to participate in the study. It was difficult to persuade patients and their relatives to give consent. The biggest limitation was of resources as this study was not externally funded so, rather than a food model, we used pictures in Powerpoint presentation. It was difficult to engage the patients for a longer period during didactic teaching so we had to complete this in 90 minutes. There was no funding for blood tests so we had to rely on the results of patients' blood tests performed by their physician. We conducted the education sessions in the institute, which was centrally located, and where we also took height and weight measurements. We found that the patients were appreciative of our work and, when contacted personally after 6 months by the lead researcher, all of them agreed to participate in the followup. Future studies should consider a whole-day course with patients bringing their own sample food to share with others and to use it for interactive discussion.

Our study has several important limitations. The refusal of some patients to participate in the educational intervention despite having previously consented to being randomised is likely to have introduced a bias into the analysis, as the groups may have differed in their attitude to education. In addition, our study employed a relatively short duration of follow-up of 6 months. Previous experience of patients randomised to the validated DESMOND structured diabetes intervention or a control group showed that there was no metabolic benefit associated with the intervention after 3 years, although some benefits persisted in terms of improved understanding of diabetes. ${ }^{21}$ Finally, the education materials were not validated formally in advance of the study. The retention of all subjects in the analysis is a strength of the study, which was possible due to recruitment of the local population and a personal approach by the investigator.

\section{Conclusions}

Provision of a single session of diabetes education to recently diagnosed type 2 diabetes patients in Nepal was associated with improvements in measures of plasma glucose 6 months later. This approach may provide a pragmatic approach to improving diabetes self-care in resource-limited countries such as Nepal.

Acknowledgements Dr Mike Gwilt (GT Communications) provided editorial assistance to the authors (no funding was involved).

Conflict of interest None.

Funding There was no external funding and the work was done as a part of JP's PhD thesis.

\section{References}

1. American Diabetes Association. 8. Obesity management for the treatment of type 2 diabetes: Standards of Medical Care in Diabetes-2019. Diabetes Care 2019;42(Suppl 1):S81-S89. https://doi.org/10.2337/dc19-S008

2. Cosentino F, Grant PJ, Aboyans V, et al. 2019 ESC guidelines on diabetes, pre-diabetes, and cardiovascular diseases developed in collaboration with the EASD. Eur Heart J 2020;41:255-323. https://doi.org/10.1093/eurheartj/ehz486

3. American Diabetes Association. 5. Lifestyle management: Standards of Medical Care in Diabetes-2019. Diabetes Care 2019;42(Suppl 1):S46-S60. https://doi.org/10.2337/dc19-S005

4. National Institute for Health and Care Excellence. Type 2 diabetes in adults: management. NICE guideline [NG28] (updated August 2019). Available at: https://www.nice.org.uk/guidance/ng28 (accessed January 2020).

5. Whittemore R, Vilar-Compte M, De La Cerda S, et al. Challenges to diabetes self-management for adults with type 2 diabetes in low-resource settings in Mexico City: a qualitative descriptive study. Int J Equity Health 2019; 18:133. https://doi.org/10.1186/s12939-019-1035-x

6. De Man J, Aweko J, Daivadanam M, et al. Diabetes self-management in three different income settings: cross-learning of barriers and opportunities. PLoS One 2019;14:e0213530. https://doi.org/10.1371/journal. pone. 0213530

7. Gyawali B, Sharma R, Neupane D, et al. Prevalence of type 2 diabetes in Nepal: a systematic review and meta-analysis from 2000 to 2014. Glob Health Action 2015;8:29088. https://doi.org/10.3402/gha.v8.29088

8. Gyawali B, Hansen MRH, Povlsen MB, et al. Awareness, prevalence, treatment, and control of type 2 diabetes in a semi-urban area of Nepal: findings from a cross-sectional study conducted as a part of COBIN-D trial. PLOS One 2018;13:e0206491. https://doi.org/10.1371/journal.pone.0206491

9. Lean MEJ, Leslie WS, Barnes AC, Brosnahan N, et al. Durability of a primary care-led weight-management intervention for remission of type 2 diabetes: 2-year results of the DiRECT open-label, cluster-randomised trial. Lancet Diabetes Endocrinol 2019;7(5):344-55. https://doi.org/10.1016/S22138587(19)30068-3

10. Thomas DE, Elliott EJ. The use of low-glycaemic index diets in diabetes control. Br J Nutr 2010;104:797-802. https://doi.org/10.1017/ S0007114510001534

11. Sigal RJ, Armstrong MJ, Colby P, et al. Physical activity and diabetes. Can J Diabetes 2013;37(Suppl 1):S40-S44. https://doi.org/10.1016/j.jcjd. 2013.01.018

12. Shrestha N, Yadav SB, Joshi AM, et al. Diabetes knowledge and associated factors among diabetes patients in central Nepal. Int J Collaborative Res Intern Med Public Health 2015:7:82-91.

13. Palmer SC, Mavridis D, Nicolucci A, et al. Comparison of clinical outcomes and adverse events associated with glucose-lowering drugs in patients with type 2 diabetes: a meta-analysis. JAMA 2016;316:313-24. https://doi.org/ 10.1001/jama.2016.9400

14. Piepoli MF, Hoes AW, Agewall S, et al. 2016 European Guidelines on cardiovascular disease prevention in clinical practice: The Sixth Joint Task Force of the European Society of Cardiology and Other Societies on Cardiovascular Disease Prevention in Clinical Practice (constituted by representatives of 10 societies and by invited experts). Developed with the special contribution of the European Association for Cardiovascular Prevention \& Rehabilitation (EACPR). Eur Heart J 2016;37:2315-81. https://doi.org/10.1093/ eurheartj/ehw106

15. Li Y, Zhao L, Yu D, Ding G. The prevalence and risk factors of dyslipidemia in different diabetic progression stages among middle-aged and elderly 
populations in China. PLoS One 2018;13:e0205709. https://doi.org/ 10.1371/journal.pone.0205709

16. Dinneen SF. Structured education for people with type 2 diabetes. BMJ 2008:336:459-60. https://doi.org/10.1136/bmj.39478.693715.80

17. Health Service Executive. Review of diabetes structured education. Republic of Ireland 2009. Available at https://www.hse.ie/eng/services/ publications/topics/diabetes/diabetesstructureded.pdf (reviewed January 2020).

18. Akhter K, Bunn C, Graffy J, et al. Empowerment-based education for established type 2 diabetes in rural England. Pract Diabetes 2017;34:83-8. https://doi.org/10.1002/pdi.2088

19. National Institute for Health and Care Excellence. Patient-education models for diabetes. Available at: https://www.nice.org.uk/guidance/TA60/ documents/assessment-report-patient-education-models-for-diabetes-2 (reviewed January 2020)

20. Chatterjee S, Davies MJ, Heller S, Speight J, Snoek FJ, Khunti K. Diabetes structured self-management education programmes: a narrative review and current innovations. Lancet Diabetes Endocrinol 2018:6(2):130-42. https://doi.org/10.1016/S2213-8587(17)30239-5

21. Khunti K, Gray LJ, Skinner T, et al. Effectiveness of a diabetes education and self-management programme (DESMOND) for people with newly diagnosed type 2 diabetes mellitus: three year follow-up of a cluster randomised controlled trial in primary care. BMJ 2012;344:e2333. https://doi.org/10.1136/bmj.e2333

\section{5th Joint Meeting of ABCD \& The Renal Association 24th-25th February, 2021}

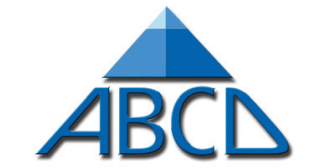

Association of British Clinical Diabetologists

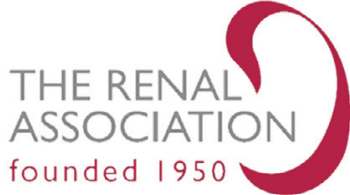

\section{Diabetes and Kidney Disease: Advances and Controversies}

This virtual meeting will be held over two evenings between 5:30 and 7:30 PM.

We will bring together renowned leaders both nationally and internationally in the field of diabetes related kidney disease to discuss current challenges, potential areas intervention and new therapeutic opportunities.

The meeting will include opportunities to consider new ways to work collaboratively across specialties and between primary and secondary care, understand how new technologies for glycaemic monitoring may benefit people on haemodialysis and consider how we improve patient engagement in our clinical settings.

We will also have a symposium looking at the latest data relating to SGLT2I and how we will be using these drugs in the coming years. Additionally we are inviting abstract submissions and presenting the best abstracts received and will be providing updates on the latest guidelines being produced by the diabetes and renal guideline writing group.

\section{Who should attend?}

- Any member of the MDT team caring for people with diabetic kidney disease

- Consultants and trainees in diabetes

- Consultants and trainees in renal disease

- General physicians and GPs with an interest in diabetes and kidney disease

- Specialist nurses in diabetes or kidney disease

- Dieticians and podiatrists with an interest in diabetic renal disease

- Anyone wishing to learn more about the challenges and opportunities relating to management of diabetes and kidney disease

\section{ABSTRACT SUBMISSIONS \\ We are inviting the submission of abstracts for posters on the subjects of diabetes and kidney disease. \\ Submitted abstracts should be of no more than 250 words and the highest ranked will be selected to be presented as posters at the meeting.}

\section{Abstract submission deadline - Monday 21 st December 2020.} Please submit your abstract via the online submission form at https://abcd.care/submit-abstract

If you have submitted an abstract, you will be notified as to whether or not your abstract has been selected for presentation as a poster at the meeting as soon as possible after the closing date. 